\title{
Identification of novel pathway partners of p68 and p72 RNA helicases through Oncomine meta-analysis Brian J Wilson and Vincent Giguère*
}

\author{
Address: Molecular Oncology Group, Room H5-45, McGill University Health Centre, 687 Pine Avenue West, Montréal, Québec, Canada \\ Email: Brian J Wilson - brian.wilson2@mcgill.ca; Vincent Giguère* - vincent.giguere@mcgill.ca \\ * Corresponding author
}

Published: 15 November 2007

BMC Genomics 2007, 8:419 doi:10.1186/147|-2164-8-419
Received: 21 June 2007

Accepted: 15 November 2007

This article is available from: http://www.biomedcentral.com/|47|-2/64/8/4I9

(c) 2007 Wilson and Giguère; licensee BioMed Central Ltd.

This is an Open Access article distributed under the terms of the Creative Commons Attribution License (http://creativecommons.org/licenses/by/2.0), which permits unrestricted use, distribution, and reproduction in any medium, provided the original work is properly cited.

\begin{abstract}
Background: The Oncomine ${ }^{\mathrm{TM}}$ database is an online collection of microarrays from various sources, usually cancer-related, and contains many "multi-arrays" (collections of analyzed microarrays, in a single study). As there are often many hundreds of tumour samples/microarrays within a single multi-array results from coexpressed genes can be analyzed, and are fully searchable. This gives a potentially significant list of coexpressed genes, which is important to define pathways in which the gene of interest is involved. However, to increase the likelihood of revealing truly significant coexpressed genes we have analyzed their frequency of occurrence over multiple studies (meta-analysis), greatly increasing the significance of results compared to those of a single study.
\end{abstract}

Results: We have used the DEAD-box proteins $p 68(D d x 5)$ and $p 72(D d x \mid 7)$ as models for this coexpression frequency analysis as there are defined functions for these proteins in splicing and transcription (known functions which we could use as a basis for quality control). Furthermore, as these proteins are highly similar, interact together, and may be to some degree functionally redundant, we then analyzed the overlap between coexpressed genes of p68 and p72. This final analysis gave us a highly significant list of coexpressed genes, clustering mainly in splicing and transcription (recapitulating their published roles), but also revealing new pathways such as cytoskeleton remodelling and protein folding. We have further tested a predicted pathway partner, RNA helicase $A(D h \times 9)$ in a reciprocal meta-analysis that identified $p 68$ and $p 72$ as being coexpressed, and further show a direct interaction of Dhx9 with p68 and p72, attesting to the predictive nature of this technique.

Conclusion: In summary we have extended the capabilities of Oncomine ${ }^{\mathrm{TM}}$ by analyzing the frequency of coexpressed genes over multiple studies, and furthermore assessing the overlap with a known pathway partner (in this case $\mathrm{p} 68$ with $\mathrm{p} 72$ ). We have shown our predictions corroborate previously published studies on $\mathrm{p} 68$ and p 72 , and that novel predictions can be easily tested. These techniques are widely applicable and should increase the quality of data from future meta-analysis studies.

\section{Background}

Recently there have been attempts to correlate published microarrays, using software that can analyze many thou- sands of microarrays at one time. One such program is called Oncomine $e^{\mathrm{TM}}[1]$, where each study within Oncomine $^{\mathrm{TM}}$ is in essence a collection of individual microarrays 
from many patient samples[2]. These "multi-arrays" usually utilise either normal or tumour biopsy samples (or compare both together), from various tissue sources.

One function of Oncomine $e^{\mathrm{TM}}$ is a search tool where the user's chosen gene is correlated in expression, within multi-arrays, with other genes in the array (both high and low expression, over all the samples in the multi-array).
For example searching p72 (DDX17) gives several correlations in many multi-arrays. Focusing within the study Whitney_normal there is a high correlation with expression of fibrillarin, over the 147 blood samples tested (Figure 1A). In samples where p72 expression was diminished, so was fibrillarin, and conversely when p72 expression was high, so is that of fibrillarin. This result is
A.

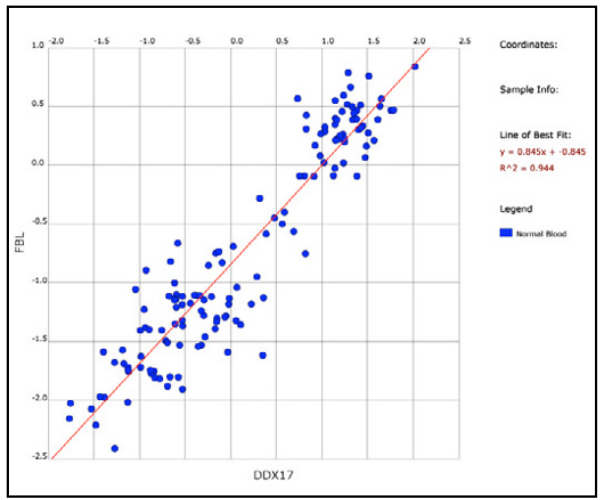

From: Whitney_normal [blood]
C.

p68 multi-arrays

Whitney_Normal (147)

Shyamsundar_Normal (123)

Hsiao Normal (62)

Ramaswamy_Multi-cancer_2 (76)

Ramaswamy_Multi-cancer (288)

Higgins_Renal (44)

Vasselli_Renal (58)

Lacayo_Leukemia (87)

Bullinger Leukemia (119)

Cario_Leukemia (51)

Dhanasekaran_Prostate (101)

Lapointe_Prostate (112)

Garber_Lung (73)

Gruvberger_Breast (58)

Sperger Others (74)

Chen_Liver (197)

Schaner_Ovarian (44)

Iacobuzio-Donahue_Pancreas_2 (31)

Lossos_Lymphoma (35) p72 multi-arrays

Whitney_Normal (147)

$10 / 19$ overlap

Shai_Brain (41)

Nutt_Brain (50)

Ramaswamy_Multi-cancer_2 (76)

Ramaswamy_Multi-cancer (288)

Boer_Renal (180)

Yu_Prostate (112)

Lacayo_Leukemia (87)

Fine_Leukemia (42)

Cario_Leukemia (51)

Dhanasekaran_Prostate (101)

Lapointe_Prostate (112)

Singh_Prostate (102)

vandeVijver_Breast (295)

Garber_Lung (73)

Lu_Ovarian (50)

Haqq_Melanoma (37)

Chen_Liver (197)

Lossos_Lymphoma (35)

B.
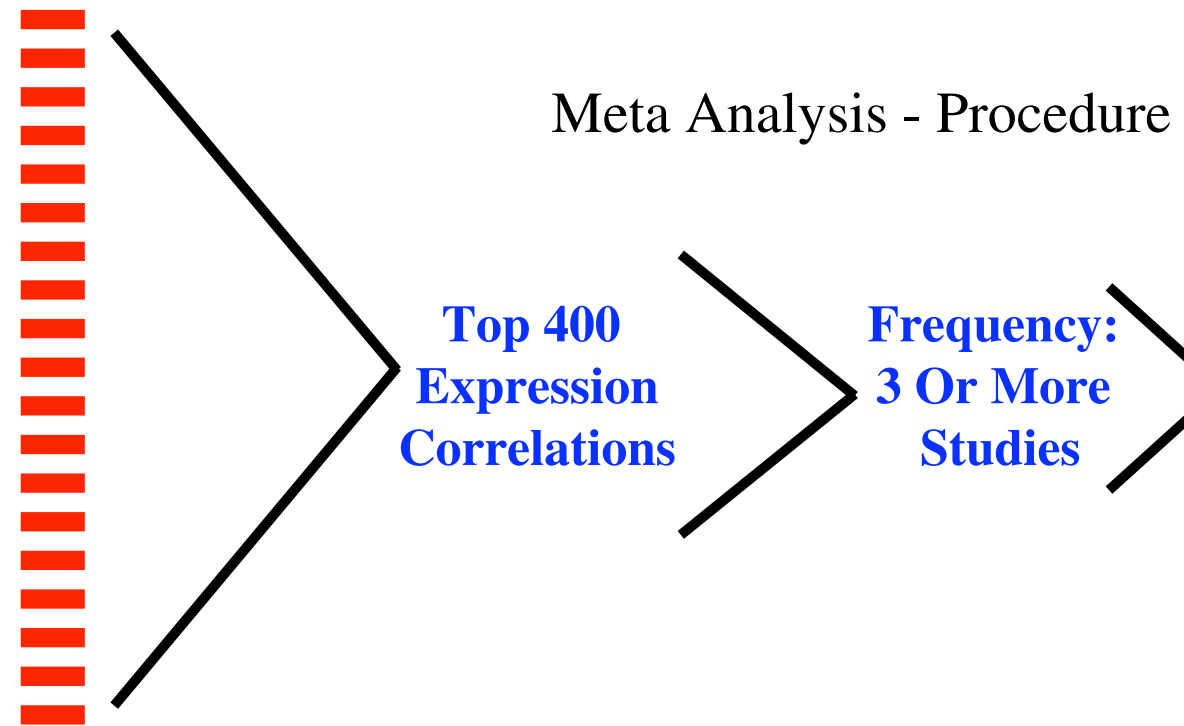

Meta Analysis - Procedure

Multi-array

\section{Figure I}

Oncomine studies utilised and methodology of analysis. (A) Screenshot example of Oncomine ${ }^{\mathrm{TM}}$ output of $\mathrm{P72}$ (DDXI 7) coexpression with fibrillarin (FBL) in one multi-array study, covering 147 samples. $p 72$ is $X$-axis and fibrillarin is $Y$-axis. (B) Procedure employed for meta-analysis of 19 different multi-arrays after searching for either p68 or p72, extracting the top 400 coexpressed genes from each multi-array, and comparing for frequency of repetition. (C) Chosen multi-arrays to be studied for both $\mathrm{p} 68$ and $\mathrm{p} 72$. 
A.
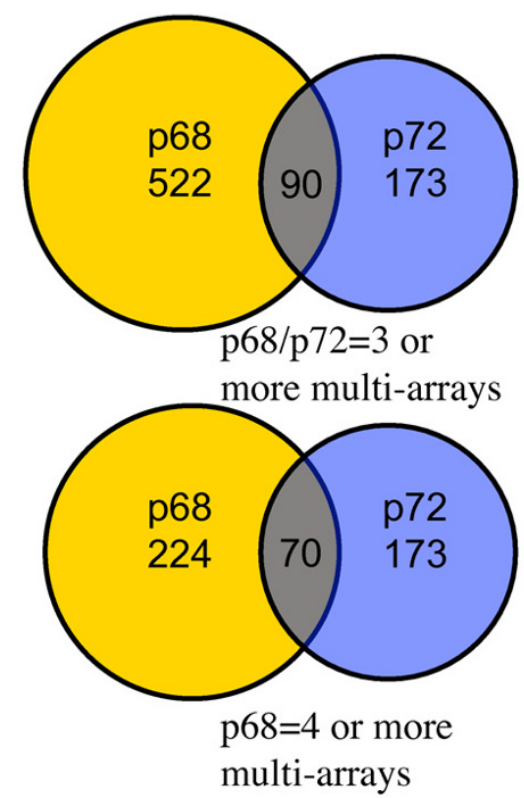

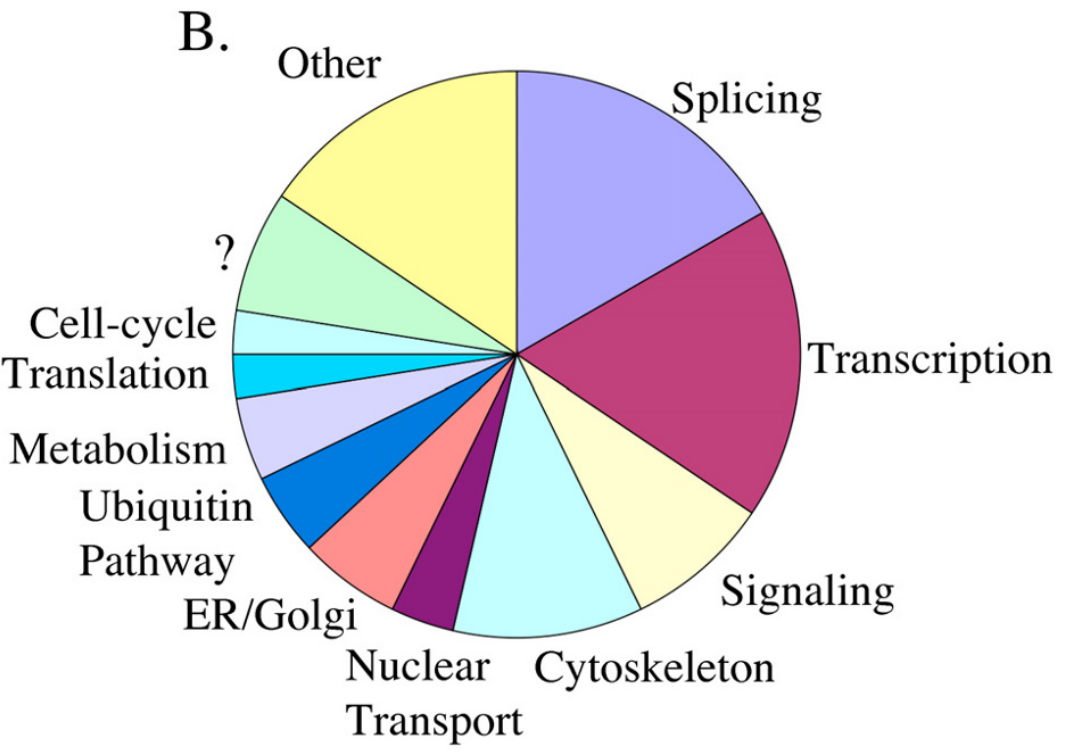

Figure 2

Analysis of overlap of p68 and p72 coexpressed genes. (A) Venn diagram of overlap of frequency $=3$ or more, genes from p68 and p72 analysis, and when p68 frequency is increased to 4 or more. (B) Ontology pie-chart of p68/p72 overlapping frequency $=3$ or more, gene products.

made more significant given that p72 and fibrillarin have previously been shown to interact together[3].

Correlations like this can show if proteins may be in the same pathway (e.g. both coregulated together, or one directly affecting the other), although it cannot show more than association. In an attempt to further increase the stringency of Oncomine ${ }^{\mathrm{TM}}$ to elude to these pathways we chose to test the DEAD-box proteins p 68 and p72 because they are highly similar proteins that interact together and have been shown to be involved in defined cellular functions including splicing and transcription, which can then be used as a quality control measure of this technique [4-10]. Also as p68 and p72 are so similar there is the possibility that they may to some extent be functionally redundant.

In total this means that we can perform a meta-analysis of p68 coexpressed genes independent to that of p72, then compare the results for overlap (Figure 1B). If the gene lists were to give a significant overlap then this would act to support the notion that the technique is highly selective. Our results reveal that, not only does this technique corroborate previously published data on p68 and p72, it also generates testable predictions of novel pathway partners of p68 and p72.

\section{Results}

Overlapping coexpressed genes of p68 and p72

Multi-arrays chosen for meta-analysis had many individual samples/microarrays, indicating that a good correlation coefficient given by Oncomine ${ }^{\mathrm{TM}}$ is already highly significant. Figure $1 \mathrm{C}$ indicates the chosen multi-array studies for p68 and p72. Note that there is almost a $50 \%$ overlap of studies chosen.

Meta-analysis results, with frequency of 3 or more, for $\mathrm{p} 68$ yielded a higher volume of hits than for p72 (see Additional file 1). Both of these lists were compared for common genes and the common list was further assessed for ontology and full gene names (Table 1). Remarkably, we observed a large number of overlapping genes, indicative of the stringency employed in this technique.

Even when the stringency was further augmented by increasing the p 68 frequency cut-off to 4 or more multiarrays (21\% and above overlap within p68 multi-arrays), this lost almost 300 p68 hits, but only reduced the number of overlapping genes with p72 from 90 to 70 (Figure $2 \mathrm{~A}$ ). The highest frequency of overlap of p68 and p72 occurred in splicing, consistent with previous reports of their role in this process. Further validation of this technique was observed by the reciprocal gene hits of p68 and 
Table I: Frequency overlap between p68 and p72 coexpressed genes.

\begin{tabular}{|c|c|c|c|c|}
\hline Gene & p68\% & p72\% & Function & Gene Name \\
\hline TIAI & $26 \%$ & $16 \%$ & Splicing & cytotoxic granule-associated RNA-binding protein \\
\hline SFRS5 & $37 \%$ & $21 \%$ & Splicing & splicing factor, arginine/serine-rich, 5 \\
\hline SFPQ & $42 \%$ & $47 \%$ & Splicing & $\begin{array}{l}\text { splicing factor proline/glutamine rich (polypyrimidine tract binding protein } \\
\text { associated) }\end{array}$ \\
\hline SFI & $37 \%$ & $26 \%$ & Splicing & splicing factor I \\
\hline MBNLI & $53 \%$ & $32 \%$ & Alternative Splicing & muscleblind-like protein(Triplet-expansion RNA-binding protein) \\
\hline HNRPHI & $47 \%$ & $32 \%$ & Splicing & heterogeneous nuclear ribonucleoprotein $\mathrm{H}$ (hnRNP H) \\
\hline CROP & $21 \%$ & $37 \%$ & SR Protein -Splicing? & cisplatin resistance-associated overexpressed protein (LUC7A) \\
\hline CPSF2 & $42 \%$ & $21 \%$ & Splicing & cleavage and polyadenylation specificity factor \\
\hline C6orflII & $32 \%$ & $32 \%$ & Splicing & splicing factor, arginine/serine-rich 130 \\
\hline FLJ 12529 & $21 \%$ & $16 \%$ & Splicing & pre-mRNA cleavage factor I, $59 \mathrm{kDa}$ subunit \\
\hline DD $\times 5$ & $100 \%$ & $26 \%$ & Splicing/Transcription & p68 DEAD-box RNA helicase \\
\hline DDXI7 & $26 \%$ & $100 \%$ & Splicing/Transcription & p72 DEAD-box RNA helicase \\
\hline PAPOLA & $26 \%$ & $21 \%$ & Transcription/Splicing & poly $(A)$ polymerase alpha \\
\hline ILF3 & $26 \%$ & $21 \%$ & Transcription/Splicing & NFARI/NF-90/subunit of NFAT transcription factor \\
\hline PNN & $16 \%$ & $21 \%$ & Transcription/Splicing & $\operatorname{pinin}(D R S)$ \\
\hline XBPI & $26 \%$ & $21 \%$ & Transcription/ER-alpha pathway & X-box binding protein I \\
\hline THRAP2 & $32 \%$ & $21 \%$ & Transcription? & thyroid hormone receptor associated protein 2 \\
\hline RORA & $26 \%$ & $26 \%$ & Transcription & RAR-related orphan receptor alpha \\
\hline PTMA & $21 \%$ & $16 \%$ & Transcription & prothymosin, alpha (gene sequence 28 ) \\
\hline $\mathrm{DH} \times 9$ & $47 \%$ & $32 \%$ & Transcription & RNA Helicase A/DEAH (Asp-Glu-Ala-His) box polypeptide 9 \\
\hline BMII & $21 \%$ & $16 \%$ & Transcription Silencing & $\begin{array}{l}\text { B lymphoma Mo-MLV insertion region (mouse) [Polycomb complex protein } \\
\text { BMI-I] }\end{array}$ \\
\hline SMARCA2 & $16 \%$ & $21 \%$ & Transcription & $\begin{array}{l}\text { SWI/SNF related, matrix associated, actin dependent regulator of chromatin, } \\
\text { subfamily a, member } 2\end{array}$ \\
\hline HIFIA & $16 \%$ & $16 \%$ & Transcription & $\begin{array}{l}\text { hypoxia-inducible factor I, alpha subunit (basic helix-loop-helix transcription } \\
\text { factor) }\end{array}$ \\
\hline MAP3K7IP2 & $26 \%$ & $21 \%$ & Signal Transduction/Transcription & mitogen-activated protein kinase kinase kinase 7 interacting protein 2 (TAB2) \\
\hline PRKARIA & $47 \%$ & $32 \%$ & Signal Transduction & $\begin{array}{l}\text { protein kinase, cAMP-dependent, regulatory, type I, alpha (tissue specific } \\
\text { extinguisher I) }\end{array}$ \\
\hline PIK3RI & $21 \%$ & $21 \%$ & Signal Transduction & phosphoinositide-3-kinase, regulatory subunit I (p85 alpha) \\
\hline HIPK2 & $32 \%$ & $16 \%$ & Signal Transduction/Apoptosis & homeodomain interacting protein kinase 2 \\
\hline DNAJC3 & $21 \%$ & $16 \%$ & Signal Transduction & $\begin{array}{l}\text { DnaJ homolog subfamily } \mathrm{C} \text { member } 3 \text { (Interferon-induced, double-stranded } \\
\text { RNA-activated protein kinase inhibitor) }\end{array}$ \\
\hline CSNKIAI & $32 \%$ & $21 \%$ & Signal Transduction & casein kinase I, alpha I \\
\hline GNAS & $21 \%$ & $21 \%$ & Receptor-Stimulated G-Protein & $\begin{array}{l}\text { guanine nucleotide binding protein (G protein), alpha stimulating activity } \\
\text { polypeptide I }\end{array}$ \\
\hline $\mathrm{AB} 12$ & $26 \%$ & $21 \%$ & Cytoskeleton & Abl-interactor 2 (Abelson interactor 2 ) \\
\hline ARPC3 & $21 \%$ & $26 \%$ & Cytoskeleton & actin related protein $2 / 3$ complex, subunit $3,21 \mathrm{kDa}$ (p2I-ARC) \\
\hline FNBP4 & $26 \%$ & $21 \%$ & Cytoskeleton? & formin binding protein 4 \\
\hline WASPIP & $16 \%$ & $16 \%$ & Cytoskeleton & Wiskott-Aldrich syndrome protein interacting protein \\
\hline UTRN & $16 \%$ & $21 \%$ & Cytoskeleton & utrophin (homologous to dystrophin) \\
\hline RAP2A & $16 \%$ & $16 \%$ & Cytoskeleton? & RAP2A, member of RAS oncogene family \\
\hline NEDD5 & $16 \%$ & $21 \%$ & Cytoskeleton/cell-cycle? & septin 2 (GTP-binding protein family) \\
\hline ACTB & $16 \%$ & $26 \%$ & Cytoskeleton & beta actin \\
\hline MAPRE2 & $16 \%$ & $16 \%$ & Cytoskeleton & microtubule-associated protein, RP/EB family, member 2 \\
\hline SDCBP & $21 \%$ & $21 \%$ & Scaffold Protein & syndecan binding protein (syntenin) \\
\hline HNRPU & $42 \%$ & $32 \%$ & Nuclear Matrix Attachment & heterogeneous nuclear ribonucleoprotein $U$ (scaffold attachment factor $A$ ) \\
\hline XPOI & $26 \%$ & $21 \%$ & Nuclear Export & exportin I (CRMI homolog, yeast) \\
\hline TNPOI & $26 \%$ & $32 \%$ & Nuclear Import & transportin I \\
\hline NUPI33 & $26 \%$ & $16 \%$ & Nuclear Pore & nuclear pore complex protein Nup 133 \\
\hline ZFR & $26 \%$ & $21 \%$ & Nuclear RNA binding & zinc finger RNA binding protein \\
\hline RAB5A & $16 \%$ & $16 \%$ & Endocytosis & RAB5A, member RAS oncogene family \\
\hline RAB6A & $68 \%$ & $16 \%$ & Golgi-ER trafficking & RAB6A, member RAS oncogene family \\
\hline GDI2 & $26 \%$ & $26 \%$ & ER-golgi?(Interacts Rab6, above) & rab GDP-dissociation inhibitor, beta \\
\hline EDEMI & $21 \%$ & $32 \%$ & Calnexin cycle/protein folding & ER degradation enhancer, mannosidase alpha-like I \\
\hline RABI4 & $32 \%$ & $26 \%$ & Golgi-endosome trafficking & RABI4, member RAS oncogene family \\
\hline PLEKHB2 & $26 \%$ & $16 \%$ & post-golgi vesicle protein & pleckstrin homology domain containing, family B (evectins) member 2 \\
\hline TMP2I & $37 \%$ & $37 \%$ & Trafficking & transmembrane trafficking protein \\
\hline TRAMI & $26 \%$ & $16 \%$ & Protein Translocation & translocation associated membrane protein I \\
\hline
\end{tabular}


Table I: Frequency overlap between p68 and p72 coexpressed genes. (Continued)

\begin{tabular}{|c|c|c|c|c|}
\hline SLC38A2 & $21 \%$ & $21 \%$ & Amino acid transport & Solute carrier family 38, member 2 \\
\hline SLC25A5 & $26 \%$ & $25 \%$ & ADP/ATP carrier protein & $\begin{array}{l}\text { Solute carrier family } 25 \text { (mitochondrial carrier; adenine nucleotide } \\
\text { translocator), member } 5\end{array}$ \\
\hline CGI-I09 & $37 \%$ & $16 \%$ & Protein transport? & hypothetical protein \\
\hline USP9X & $21 \%$ & $16 \%$ & Ubiquitin & ubiquitin specific protease $9, X$ chromosome (Drosophila fat facets related) \\
\hline UBE2 $J I$ & $21 \%$ & $32 \%$ & Ubiquitin & ubiquitin-conjugating enzyme E2, JI (UBC6 homolog, yeast) \\
\hline UBE3A & $16 \%$ & $21 \%$ & Ubiquitin & ubiquitin protein ligase $\mathrm{E} 3 \mathrm{~A}$ \\
\hline BIRC6 & $16 \%$ & $21 \%$ & Ubiquitin ligase/Anti-apoptosis & baculoviral IAP repeat-containing 6 (apollon) \\
\hline BIRC2 & $32 \%$ & $21 \%$ & Apoptosis-resistance & baculoviral IAP repeat-containing 2 \\
\hline PSMA2 & $21 \%$ & $21 \%$ & Proteasome & proteasome (prosome, macropain) subunit, alpha type, 2 \\
\hline PIASI & $26 \%$ & $21 \%$ & E3-SUMO Ligase & protein inhibitor of activated STAT, I (DEAD/H box-binding protein I) \\
\hline MAK3 & $21 \%$ & $16 \%$ & $\mathrm{~N}$-acetyltransferase & Mak3 homolog (S. cerevisiae) \\
\hline PFAAP5 & $21 \%$ & $21 \%$ & Immune? & phosphonoformate immuno-associated protein 5 \\
\hline MCP & $32 \%$ & $26 \%$ & Immune & $\begin{array}{l}\text { membrane cofactor protein (CD46, trophoblast-lymphocyte cross-reactive } \\
\text { antigen) }\end{array}$ \\
\hline SMBP & $26 \%$ & $16 \%$ & Membrane protein & SM-I I044 binding protein \\
\hline MKLNI & $21 \%$ & $21 \%$ & Ischemic tolerance/Cell adhesion? & muskelin I, intracellular mediator containing kelch motifs \\
\hline ALDOA & $26 \%$ & $16 \%$ & Metabolism (glycolysis) & aldolase $\mathrm{A}$, fructose-bisphosphate \\
\hline IDII & $32 \%$ & $26 \%$ & Metabolism & isopentenyl-diphosphate delta isomerase \\
\hline CYB5-M & $26 \%$ & $16 \%$ & Metabolism & cytochrome b5 outer mitochondrial membrane precursor \\
\hline GLOI & $37 \%$ & $21 \%$ & $\begin{array}{l}\text { Metalloglutathione }(\mathrm{GSH}) \\
\text { transferase }\end{array}$ & glyoxalase I \\
\hline EIF3S6 & $21 \%$ & $16 \%$ & Translation & eukaryotic translation initiation factor 3 , subunit $648 \mathrm{kDa}$ \\
\hline EIFIAX & $16 \%$ & $21 \%$ & Translation & eukaryotic translation initiation factor IA \\
\hline PCBP2 & $16 \%$ & $16 \%$ & Translation & poly $(\mathrm{rC})$ binding protein 2 (hnRNPE2) \\
\hline HNRPA2BI & $32 \%$ & $21 \%$ & Cell proliferation? & heterogeneous nuclear ribonucleoprotein $A 2 / B$ I \\
\hline CDK6 & $16 \%$ & $16 \%$ & Cell-cycle & cyclin-dependent kinase 6 \\
\hline CCNE2 & $16 \%$ & $16 \%$ & Cell-cycle & GI/S-specific cyclin E2 \\
\hline PUM2 & $26 \%$ & $21 \%$ & Meiosis/RNA-binding & pumilio homolog 2 (Drosophila) \\
\hline TRA2A & $16 \%$ & $26 \%$ & RNA-binding/? & transformer-2 alpha (putative MAPK activating protein PM24) \\
\hline ATXN2 & $16 \%$ & $21 \%$ & ? (but has RNA motif) & ataxin 2 \\
\hline GTF2IPI & $21 \%$ & $26 \%$ & Pseudogene & general transcription factor II, i, pseudogene I \\
\hline $\mathrm{H} 4 \mathrm{I}$ & $53 \%$ & $26 \%$ & ? & hypothetical protein \\
\hline Cl9orfl3 & $26 \%$ & $37 \%$ & ? & family with sequence similarity $6 \mathrm{I}$, member $A(F A M 6 \mid A)$ \\
\hline $\mathrm{CNIH}$ & $26 \%$ & $26 \%$ & ? & cornichon homolog (TGAM77) \\
\hline LOC400986 & $26 \%$ & $26 \%$ & ? & protein immuno-reactive with anti-PTH polyclonal antibodies (HEMI) \\
\hline ANKRDI7 & $21 \%$ & $21 \%$ & ? & ankyrin repeat domain 17 (breast cancer antigen NY-BR-16) \\
\hline RHOBTB3 & $16 \%$ & $16 \%$ & ? (GTPase) & Rho-related BTB domain containing 3 \\
\hline
\end{tabular}

90 genes were identified to be both coexpressed with p68 and p72, and are arranged by function. For clarity all coexpressed gene products with a $30 \%$ or greater coexpression frequency correlation for either $p 68$ or $p 72$ are in bold.

p72 (i.e. p72 was a positive for p68 and vice-versa), again consistent with their interaction within the same pathways.

The next most abundant function of p68 and p72 appeared to be in transcription (Figure 2B), once more consistent with previous reports. This is especially interesting given that p68 and p72 were previously shown to act as coactivators for the nuclear receptor estrogen receptor $\alpha(\mathrm{ER} \alpha)$ transcription factor, and we have identified Xbox binding protein 1 (XBP1), associated with the ER $\alpha$ pathway. We have also identified 2 other nuclear receptor pathway proteins, the thyroid hormone receptor associated protein 2 (THRAP2) and the retinoic acid receptorrelated orphan receptor $\alpha(R O R A)$ transcription factor.

\section{RNA Helicase $A(D h \times 9)$ coexpresses and interacts with $p 68$ and $\mathbf{p 7 2}$}

A further interesting transcription-associated gene identified was RNA helicase A (DHX9), a member of a similar protein family to p68 and p72, all of which have been shown to interact with $\mathrm{p} 300 / \mathrm{CBP}$ coactivators[6,11-13]. The frequency for both p68 and p72 were observed to be high for RNA helicase A (almost 50\% of multi-arrays for p68, and over $30 \%$ for p72).

For this reason a similar coexpression analysis was separately performed for DHX9. Surprisingly, not only were p68 and p72 reciprocally coregulated with DHX9, but over $50 \%$ of the p68:p72 overlapped positives were also coexpressed with DHX9 (47 out of 90 - see Additional file 
2). This was powerful evidence linking Dhx9, p68 and p72 to similar pathways.

As this overlap was so high it was possible that p68 and p72 were functioning in the same complex as Dhx9. This was tested experimentally in HEK293 cells. With immunoprecipitation of either transiently transfected p68 or p72 we observed a clear interaction with endogenous Dhx9 (figure 3A). Further imunoprecipitations of endogenous p68 and p72 from lysate of mouse liver confirmed the interaction with Dhx9 (figure 3B). This was performed after incubation with RNaseA, indicating a protein:pro- tein interaction (as p68/p72/Dhx9 can all bind RNA). In the liver extract p68 and p72 also strongly immunoprecipitated a protein of $100 \mathrm{kDa}$, recognised by the Dhx9 antibody (figure 3B). It currently remains unclear if this is a different isoform of Dhx9 or a cross-reacting protein.

Altogether, these data both supported the hypothesis of p68/p72/Dhx9 existing within the same complex, and further acted as strong evidence of the predictive capabilities of the Oncomine ${ }^{\mathrm{TM}}$ analysis technique described here.
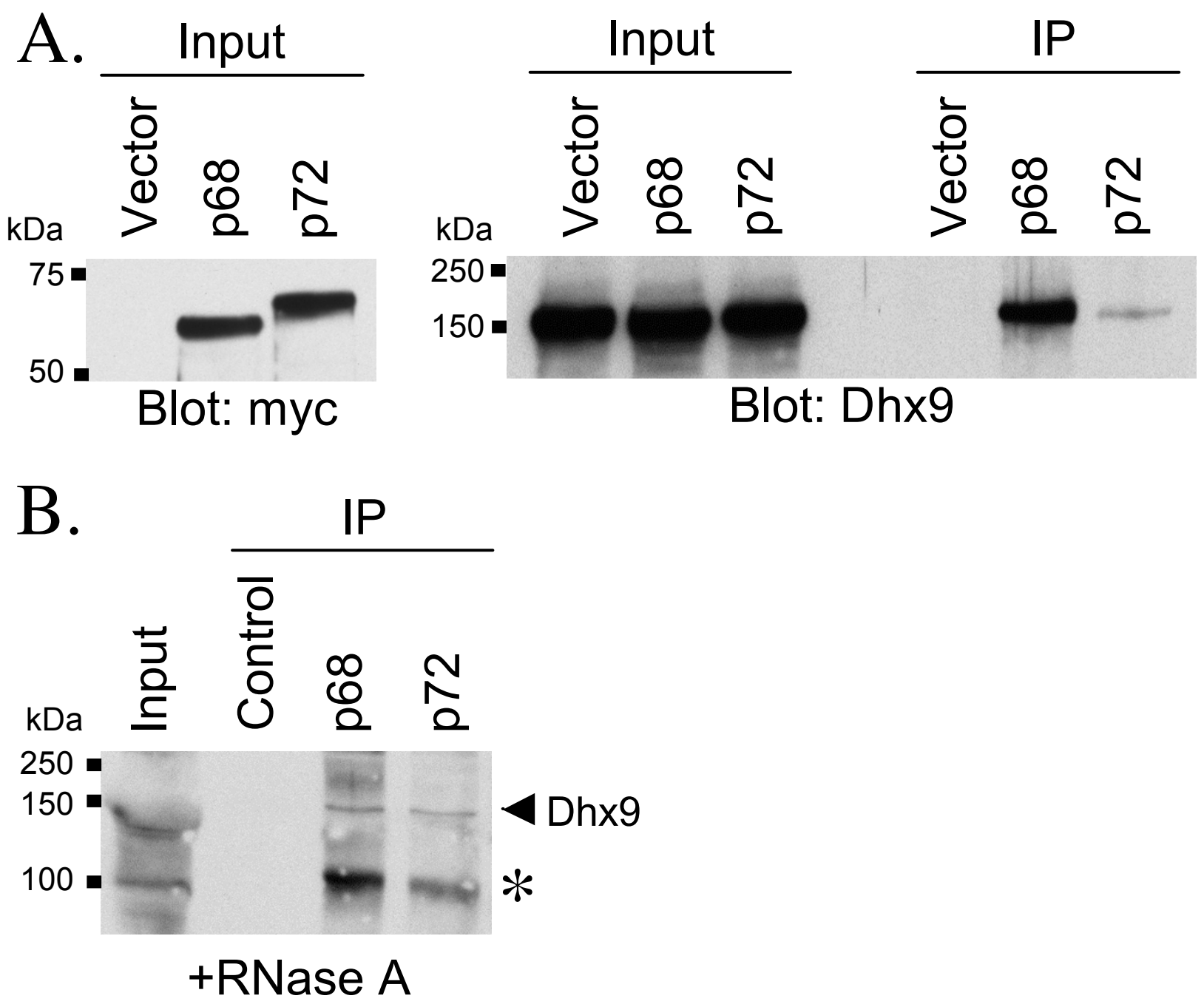

Figure 3

p68 and p72 interact directly with predicted pathway partner Dhx9. (A) Left panel shows myc immunoblot of inputs from transiently transfected myc-p68 or -p72, or vector alone. Right panel shows RNA helicase A (Dhx9) immunoblot of inputs and myc immunoprecipitations (IP). (B) Dhx9 immunoblot of endogenous IP of p68 and p72 from mouse liver lysate (RNase A pre-treated). * Indicates either a shorter Dhx9 isoform or a cross-reacting (but immuno-precipitating) protein. 


\section{Other coexpressed genes of p68 and p72}

Interestingly, there were 4 overlapped hits in the ubiquitin pathway (and one proteasome) which may be related to the observation that p 68 is highly ubiquitinated in colon cancers[14]. p68 was also recently shown to be SUMO modified, specifically SUMO-2 by PIAS1 ligase[15]. Here we shown that PIAS1 is coexpressed with p68/p72, and SUMO-2 is coexpressed with p68.

p68/p72 have also recently been shown to interact in a complex with ILF3, hnRNPU, and hnRNPH1 for microRNA processing[16]. Here, these gene products are also shown to be highly coexpressed with p68 and p72, supporting their role in the same complex/pathway (furthermore DDX3X is identified here with p68 and is also part of this microRNA processing complex).

In a separate study a group of proteins were identified in an mRNP complex with p68 and are here shown to be coexpressed with p68/p72 (SFRS5, NFAR/ILF3, HNRNPA2/B1, HNRPU, PNN, TRA2A, DDX3X) [17].

A new role for p68 and p72, suggested by our meta-analysis, might be in nuclear transport, given that a member of nuclear pore complex (Nup133) as well as nuclear import (transportin1) and export (exportin1) genes were identified as coexpressed genes.

Furthermore, coexpressed genes presented here are not limited to nuclear processes given that several cytoskeletal proteins are identified in the screening, implicating p68 and p72 in these processes (although probably indirectly as p68 and p72 are predominantly nuclear, perhaps acting via transcription or splicing). This is also true for endoplasmic reticulum (ER) or golgi proteins. Indeed, the $R A B 6 A$ trafficking protein had the highest frequency overlap for p68 (almost 70\% overlap), while being one of the lowest for p72 (16\% overlap), possibly indicative of a functional difference between both. The family member $R A B 14$ was also identified for both.

A further significant group of genes identified were involved in signal transduction, and may provide a start into analysis of regulation of p68 and p72 (although a meta-analysis like this can identify frequency of coexpression, it is impossible to say which protein may be regulating another, or indeed if both are targets of another protein).

Altogether the results of the overlapping coexpressed genes not only reiterate previous studies with either p68/ p72 but predict new potential pathways in which p68/p72 may act.

\section{Selected non-overlapping coexpressed genes of p68 and p72}

While p68 and p72 may be highly similar and involved in the same pathways, it remains likely that they are also involved in subtly different pathways. For this reason a similar ontology analysis was performed on genes that do not overlap between p68 and p72. However, given the extensive nature of the gene hits we selected all genes with frequency overlap above $30 \%$, as well as some genes of interest from lower frequencies (Table 2).

For p68 the genes above $30 \%$ generally fell into the same categories as previously, while there was only 1 gene identified for $\mathrm{p} 72$, with no obvious molecular function. Ofcourse the selected genes below 30\% were chosen based on interest and common ontological groupings, and may not be representative. However, we note that for p68 more RAB family members are identified (RAB1A, RAB11B) as well as more ER proteins, particularly protein folding chaperones (Tapasin, Calnexin, Calreticulin).

With regard to transcription, p68 coexpressed with ELK3 and HDAC2 transcriptional repressors, while p72 coexpressed with CTBP1 and HDAC7 repressors. This might be relevant given that $\mathrm{p} 68$ and $\mathrm{p} 72$ have been shown to act as transcriptional repressors, hypothesised to have different mechanisms of action as they act in a promoter-specific manner[7]. However it has been shown that CTBP1 repressive function is antagonized by pinin[18], and here, both p68 and p72 also coexpress with pinin (PNN)[17]. p68 has also been shown to be involved in p53 coactivation[4], and here we identify a coexpressed p53 coactivator hnRNPK[19] for p68/p72 and the p53-induced protein 7 (LITAF), for p68. For other transcription roles for p68 there were more nuclear receptor pathway proteins including thyroid receptor interacting protein 8 (JMJD1C),THRAP1 (THRAP2 was identified above for both p68 and p72), estrogen receptor binding protein $(E R B P)$, and the retinoic acid receptor alpha (RARA) transcription factor. p72 coexpressed with the ER-alpha repressor MTA1. We have also observed that p68 coexpressed gene ZNF9 is in the same pathway as p68/p72 coexpressed $M B N L 1$, implicated in myotonic dystrophy[20].

For p72 we note that NonO (p54nrb) has been shown to interact with SFPQ/PSF[21] (SFPQ identified as coexpressed for both p68 and p72). Furthermore EDD (a ubiquitin E3 ligase), also identified here with p72, has been shown in a complex with SFPQ[22]. Remarkably p68 has also very recently been shown to interact in a complex with NonO and SFPQ/PSF[23], again confirming the validity of the technique described here. 
Table 2: p68 and p72 frequency analysis of non-overlapping hits (all over $30 \%$ and selected below $30 \%$ ).

\begin{tabular}{|c|c|c|c|}
\hline \multicolumn{4}{|c|}{ p68 selected genes with no $p 72$ overlap } \\
\hline Gene & \% Overlap & Function & Gene Name \\
\hline FXRI & $42 \%$ & RNA-binding/Unknown & fragile $X$ mental retardation, autosomal homolog I \\
\hline HNRPK & $37 \%$ & Transcription/Translation/Signaling & heterogeneous nuclear ribonucleoprotein $\mathrm{K}$ \\
\hline NAPILI & $32 \%$ & Transcription & nucleosome assembly protein I-like I \\
\hline JMJDIC & $32 \%$ & Transcription & $\begin{array}{l}\text { jumonji domain containing protein IC (Thyroid receptor interacting } \\
\text { protein 8) }\end{array}$ \\
\hline SFRSII & $32 \%$ & Splicing & splicing factor, arginine/serine-rich II \\
\hline MAPREI & $37 \%$ & Cytoskeleton & microtubule-associated protein, RP/EB family, member I \\
\hline ACTG2 & $32 \%$ & Cytoskeleton & actin, gamma 2 , smooth muscle, enteric \\
\hline PTPNII & $32 \%$ & Signal Transduction & $\begin{array}{l}\text { protein tyrosine phosphatase, non-receptor type II (Noonan syndrome } \\
\text { I) }\end{array}$ \\
\hline JAKI & $32 \%$ & Signal Transduction & janus kinase I (a protein tyrosine kinase) \\
\hline ARF3 & $32 \%$ & Vesicular Trafficking & ADP-ribosylation factor 3 \\
\hline ANXA7 & $32 \%$ & ER-calcium mobilization & annexin A7 (Annexin VII) (Synexin) \\
\hline COX7A2L & $32 \%$ & Metabolism & cytochrome c oxidase subunit Vlla polypeptide 2 like \\
\hline C6orf55 & $32 \%$ & Anti-metastatic protein & $\begin{array}{l}\text { protein C6orf55 (Dopamine responsive protein DRG-I) (My0I2 } \\
\text { protein) }\end{array}$ \\
\hline LAPTM4A & $32 \%$ & compartmentalization of amphipathic solutes & lysosomal-associated protein transmembrane 4 alpha \\
\hline ZNF9 & $32 \%$ & $?$ & zinc finger protein 9 (a cellular retroviral nucleic acid binding protein) \\
\hline TDEI & $32 \%$ & ? & tumor differentially expressed I \\
\hline SYPL & $32 \%$ & ? & synaptophysin-like I \\
\hline NUCKS & $32 \%$ & ? & nuclear, casein kinase and cyclin-dependant kinase substrate \\
\hline ELK3 & $26 \%$ & Transcription & ELK3, ETS-domain protein (SRF accessory protein 2) \\
\hline THRAPI & $21 \%$ & Transcription & thyroid hormone receptor associated protein I \\
\hline RBBP4 & $21 \%$ & Transcription & $\begin{array}{l}\text { retinoblastoma binding protein } 4 \text { (chromatin assembly factor/CAF-I p48 } \\
\text { subunit) }\end{array}$ \\
\hline ERBP & $21 \%$ & Transcription & estrogen receptor binding protein \\
\hline RARA & $16 \%$ & Transcription & retinoic acid receptor, alpha \\
\hline HDAC2 & $16 \%$ & Transcription & histone deacetylase 2 \\
\hline SNRPB & $26 \%$ & Splicing & small nuclear ribonucleoprotein polypeptides B and BI \\
\hline TAPBP & $26 \%$ & ER chaperone/Protein folding & TAP binding protein (tapasin) \\
\hline CALR & $21 \%$ & ER chaperone/Protein folding & calreticulin \\
\hline CANX & $16 \%$ & ER Chaperone/Protein folding & calnexin \\
\hline RABIA & $26 \%$ & ER-golgi Transport & RABIA, member RAS oncogene family \\
\hline RABIIB & $21 \%$ & Membrane recycling & RABIIB, member RAS oncogene family \\
\hline UCHLI & $21 \%$ & Ubiquitin & ubiquitin carboxyl-terminal esterase LI (ubiquitin thiolesterase) \\
\hline PSMA2 & $21 \%$ & Proteolysis & proteasome (prosome, macropain) subunit, alpha type, 2 \\
\hline $\begin{array}{l}\text { PRKWNK } \\
\text { I }\end{array}$ & $21 \%$ & Proteolysis & proteasome (prosome, macropain) $26 \mathrm{~S}$ subunit, non-ATPase, I \\
\hline SUMO2 & $16 \%$ & SUMO pathway & small ubiquitin-like modifier, 2 \\
\hline CDC42 & $21 \%$ & Cell-cycle & cell division cycle 42 (GTP binding protein, 25kDa) \\
\hline CDC40 & $21 \%$ & Cell-cycle & cell division cycle 40 homolog (yeast) [pre-mRNA splicing factor 17] \\
\hline CDCIO & $26 \%$ & Cytokinesis? & septin-7 (CDCI0 protein homolog) \\
\hline LITAF & $26 \%$ & p53-induced Apoptosis & lipopolysaccharide-induced TNF factor (p53-induced protein 7) \\
\hline EIF3SIO & $26 \%$ & Translation & eukaryotic translation initiation factor 3 , subunit 10 theta, $150 / 170 \mathrm{kDa}$ \\
\hline \multicolumn{4}{|c|}{ p72 selected genes with no p68 overlap } \\
\hline Gene & \% Overlap & Function & Gene Name \\
\hline TTC3 & $32 \%$ & $?$ & tetratricopeptide repeat domain 3 \\
\hline HMGN4 & $26 \%$ & Transcription & high mobility group nucleosomal binding domain 4 \\
\hline CTBPI & $21 \%$ & Transcription/corepressor & C-terminal binding protein I \\
\hline MTAI & $21 \%$ & Transcription/ER-alpha repressor & metastasis associated I \\
\hline HDAC7A & $16 \%$ & Transcription & histone deacetylase 7A \\
\hline NONO & $16 \%$ & Splicing/Transcription & non-POU domain containing, octamer-binding ( $\mathrm{p} 54 \mathrm{nrb}$ ) \\
\hline SFRS3 & $16 \%$ & Splicing & splicing factor, arginine/serine-rich 3 \\
\hline MAP2K3 & $26 \%$ & Signal Transduction & mitogen-activated protein kinase kinase 3 \\
\hline ERBB3 & $16 \%$ & Signal Transduction & receptor protein-tyrosine kinase erbB-3 \\
\hline CSK & $16 \%$ & Signal Transduction & c-src tyrosine kinase \\
\hline CALM2 & $16 \%$ & Signal Transduction & calmodulin 2 (phosphorylase kinase, delta) \\
\hline RPS6 & $21 \%$ & Ribosome & $40 S$ ribosomal protein $\mathrm{S} 6$ \\
\hline
\end{tabular}


Table 2: p68 and p72 frequency analysis of non-overlapping hits (all over $\mathbf{3 0} \%$ and selected below $\mathbf{3 0} \%$ ). (Continued)

\begin{tabular}{llll}
\hline RPSI5A & $21 \%$ & Ribosome & 40S ribosomal protein SI5a \\
MRPS6 & $26 \%$ & Mitochondrial Ribosome Protein & mitochondrial ribosomal protein S6 \\
PABPCI & $26 \%$ & Translation & poly $(\mathrm{A})$ binding protein, cytoplasmic I \\
EIF5 & $21 \%$ & Translation & eukaryotic translation initiation factor 5 \\
EDD & $26 \%$ & ubiquitin E3 Ligase & ubiquitin--protein ligase EDD \\
ARPC3 & $26 \%$ & Cytoskeleton & actin related protein $2 / 3$ complex, subunit 3,2I kDa \\
WSBI & $21 \%$ & $?$ & WD repeat and SOCS box-containing I \\
GARNLI & $21 \%$ & $?$ & GTPase activating RANGAP domain-like I \\
?-Genes with unknown function. Genes with $>30 \%$ frequency overlap are in bold.
\end{tabular}

All coexpressed but non-overlapping gene products for $p 68$ and p72 over $30 \%$ frequency are shown (and are in bold). Selected coexpressed gene products below $30 \%$ are shown and were chosen based on interest and common ontology groups.

\section{Discussion}

The technique described here has proven useful in increasing the stringency of Oncomine ${ }^{\mathrm{TM}}$ meta-analysis, and will prove to be widely applicable. Generally individual gene levels cannot be compared from one study to another, but the strength of our analysis is an inter-study comparison (meta-analysis) after an intra-study Oncomine ${ }^{\mathrm{TM}}$ analysis (coexpression gene search).

While we still retain the strongest 400 coexpressed genes from each multi-array, it becomes de-sorted when analyzing for frequency over different studies. An example is EDEM1 (involved in protein folding in the ER), which is consistently one of the strongest correlated genes with p72, while having only a $32 \%$ frequency overlap. The same is true for p68 and Sp3 transcription factor with a frequency overlap of $37 \%$, and very highly coexpressed in these individual studies. Conversely, the technique described here is useful for comparison of coexpressed genes which may not always have a high coexpression coefficient, giving another advantage over analysis of single studies.

An interesting exception is $R A B 6 A$ with p 68 which has both the highest frequency overlap with p68 (68\%) and is almost always within the first 100 genes coexpressed with p68 in individual multi-array studies. A further exception is RNA helicase A (DHX9) which again has a high frequency of overlap with p68 (47\%) and usually is within the first 50 coexpressed genes with p68. We have also shown here for the first time an interaction by immunoprecipitation of p68 (and also p72), with Dhx9.

Furthermore, the technique described here is most useful in clustering specific genes involved in pathways when meta-analysis hits from known interacting proteins can be overlapped. We observed with our example of p68 and p72 that the overlapping hits mainly clustered into the classes of ontology in which p68/p72 had already been reported, namely splicing and transcription, further acting as validation for this type of analysis.
While some new proposed pathways for p68/p72 cannot be through direct action (e.g. cytoskeletal remodelling or ER-protein folding) it remains possible that p68/p72 are involved in these pathways indirectly via splicing/transcription/controlling nuclear shuttling. We were encouraged by the fact that p68 and/or p72 coexpressed with previously published interacting proteins such as oneanother, ILF3, hnRNPH1, hnRNPU, hnRNPA2/B1, SFRS5, Ddx3X, PIAS1, SUMO2, pinin, NonO and SFPQ and were further encouraged by observation of coexpression with members of pathways in which they were previously shown to act, such as estrogen receptor pathway (XBP1, MTA1, ERBP, DDX5, DDX17), ubiquitin pathway (USPX9, UBE2J1, UBE3A, BIRC6, UCHL1, EDD), translation (EIF3S6, EIF1A, EIF3S10, PABPC1, EIF5), and transcriptional repression (HDAC2, HDAC7A, PNN, ELK3, CTBP1, MTA1).

There also seems to be a more general role for p68 and p72 in nuclear receptor transcription pathways than first assumed (ER $\alpha$ pathway as above), for example JMJD1C, THRAP1, THRAP2, RARA, RORA, all coexpress with p68 and/or p72.

While it is clear that we have obtained a highly stringent list of potential pathway partners of p68 and p72, with regard to separable functions (i.e. non-overlapping genes of p68 and p72) we cannot say with confidence as genes generally clustered into the same pathways as for the overlapping list. This may be due to a high false-negative rate of this technique as we have used several levels of stringency, and will most likely exclude many true pathway partners of p68 and p72. However, this cost is offset by high quality results using our rigorous analysis.

\section{Conclusion}

It is apparent that we have increased the scope of the Oncomine $^{\mathrm{TM}}$ database, by utilising frequency of coexpression (meta-analysis) over different multi-array studies to predict pathway partners of searched proteins. With regard to the p68 and p72 RNA helicases we have identi- 
fied a non-exhaustive list of gene products that are likely to be present in various pathways in which p68 and/or p72 act, both corroborating previous studies and making novel predictions. For one of these, RNA helicase $\mathrm{A}(\mathrm{Dhx} 9)$, we have shown there is a direct interaction with p68 and p72. Future experimental studies using this list as a reference point will reveal the validity of this technique.

\section{Methods}

\section{Oncomine analysis}

The following procedure was undertaken for meta-analysis (figure 1B):

(1) Oncomine ${ }^{\mathrm{TM}}$ expression correlations were searched for p68 (DDX5) or p72(DDX17). (2) 19 different mult-arrays were chosen and the first 400 correlated genes within each multi-array were compared using Microsoft Excel, (separately for p68 and p72). Importantly, repetitive genes were then removed within each study, leaving only 1 representative per multi-array study. When a coregulated gene appeared in more than 3 multi-array experiments it was accepted as significant ( $3=16 \%$ frequency of the 19 ). These genes were taken as more significant than analysis of a single Oncomine ${ }^{\mathrm{Tm}}$ output. Furthermore, given that the user cannot choose which multi-array will be given by Oncomine $^{\mathrm{TM}}$ there was no attempt to specifiy different tissue types or cancer types. This had the advantage of giving a more generalised result of which pathways the proteins may be involved in, which was preferred for an initial study such as that performed here. (3) These sorted lists of coregulated genes given for p68 and p72 were compared for overlapping genes which added another level of stringency, and greatly increased the significance of the results. The genes listed were then investigated for ontology, and full gene/gene-product names, using a combination of Pubmed searches[24], Fatigo[25], and Genecards[26].

\section{Cell culture, transfection, immunoprecipitation and western blot}

HEK293 cells were transfected with either pSG5-myc, pSG5-myc-p68, pSG5-myc-p72 (plasmids were a gift from Frances Fuller-Pace, Dundee, UK), using FuGENE 6 (Roche). $48 \mathrm{~h}$ post-transfection cells were harvested on ice in buffer B $(150 \mathrm{mM} \mathrm{KCl}, 0.1 \% \mathrm{NP}-40,20 \mathrm{mM}$ Tris-HCl pH8.0, $5 \mathrm{mM} \mathrm{MgCl}_{2}, 10 \%$ glycerol, $5 \mathrm{mM} \mathrm{NaF}, 1 \times$ Roche complete protease inhibitor cocktail). $600 \mu \mathrm{g}$ of total cell extract was incubated with $5 \mu \mathrm{g} 9 \mathrm{E} 10$ anti-myc monoclonal antibody, and protein G sepharose (GE Healthcare), rotating at $4{ }^{\circ} \mathrm{C}$ for $2 \mathrm{~h}$. Pellet was washed $3 \times$ in buffer $\mathrm{B}$, boiled in protein loading buffer that was then run on an SDS-PAGE gel, transferred to pvdf and immunoblotted overnight at $4{ }^{\circ} \mathrm{C}$ for Dhx9 (Bethyl Laboratories) or myc.
For endogenous co-immunoprecipitation liver was extracted from a $3 \mathrm{mth}$ old male mouse and homogenised in buffer B (Brinkmann polytron). Lysis was allowed to happen, rotating at $4^{\circ} \mathrm{C}$ for $30 \mathrm{~min}$. Sample was then centrifuged to remove debris and further incubated with RNaseA, rotating at $4^{\circ} \mathrm{C}$ for 30 additional minutes, while preclearing lysate with protein $\mathrm{G}$ sepharose. $2 \mathrm{mg}$ of this lysate was used with $3 \mu \mathrm{g}$ of either p68 or p72 antibodies (Bethyl Laboratories) per immunoprecipitation, which were performed as above.

\section{Authors' contributions}

BJW conceived and designed the study, analyzed the data, performed the co-immunoprecipitation experiments, and wrote the manuscript. VG critically reviewed the manuscript and approved the final version.

\section{Additional material}

\section{Additional file 1}

p68(DDX5) and p72(DDX17) coexpressed genes. Table of all coexpressed genes of $p 68$ and $p 72$ (individual analyses) with frequency cutoff of 3 multi-array studies.

Click here for file

[http://www.biomedcentral.com/content/supplementary/1471-

2164-8-419-S1.xls]

\section{Additional file 2}

DHX9 coexpressed genes and overlaps with p68(DDX5) and p72(DDX17). Table of DHX9 Oncomine meta-analysis for coexpressed genes. Frequency cutoff of 3 multi-array studies. Overlap with $p 68$ and p72 individual coexpression gene lists is shown, as is the overlap with the p68:p72 common gene list.

Click here for file

[http://www.biomedcentral.com/content/supplementary/14712164-8-419-S2.xls]

\section{Acknowledgements}

We thank Catherine Dufour for harvesting of the mouse liver, and Frances Fuller-Pace for myc expression plasmids. Financial support was provided by the Canadian Institutes for Health Research, CIHR, (VG) and a McGill University Health Centre Fellowship (BJW).

\section{References}

I. Oncomine [http://www.oncomine.org]

2. Rhodes DR, Kalyana-Sundaram S, Mahavisno V, Barrette TR, Ghosh $D$, Chinnaiyan AM: Mining for regulatory programs in the cancer transcriptome. Nat Genet 2005, 37(6):579-583.

3. Ogilvie VC, Wilson BJ, Nicol SM, Morrice NA, Saunders LR, Barber GN, Fuller-Pace FV: The highly related DEAD box RNA helicases p68 and p72 exist as heterodimers in cells. Nucleic Acids Res 2003, 3 I(5): | 470- |480.

4. Bates GJ, Nicol SM, Wilson BJ, Jacobs AM, Bourdon JC, Wardrop J, Gregory DJ, Lane DP, Perkins ND, Fuller-Pace FV: The DEAD box protein p68: a novel transcriptional coactivator of the p53 tumour suppressor. Embo J 2005, 24(3):543-553.

5. Metivier R, Penot G, Hubner MR, Reid G, Brand H, Kos M, Gannon $F$ : Estrogen receptor-alpha directs ordered, cyclical, and combinatorial recruitment of cofactors on a natural target promoter. Cell 2003, I I5(6):75I-763. 
6. Watanabe M, Yanagisawa J, Kitagawa H, Takeyama K, Ogawa S, Arao Y, Suzawa M, Kobayashi Y, Yano T, Yoshikawa $H$, et al.: A subfamily of RNA-binding DEAD-box proteins acts as an estrogen receptor alpha coactivator through the $\mathbf{N}$-terminal activation domain (AF-I) with an RNA coactivator, SRA. Embo J 200I, 20(6): $|34|-\mid 352$.

7. Wilson B], Bates GJ, Nicol SM, Gregory DJ, Perkins ND, Fuller-Pace FV: The p68 and p72 DEAD box RNA helicases interact with $\mathrm{HDACI}$ and repress transcription in a promoter-specific manner. BMC Mol Biol 2004, 5: II.

8. Guil S, Gattoni R, Carrascal M, Abian J, Stevenin J, Bach-Elias M: Roles of hnRNP AI, SR proteins, and p68 helicase in c-H-ras alternative splicing regulation. Mol Cell Biol 2003, 23(8):2927-294I.

9. Honig A, Auboeuf D, Parker MM, O'Malley BW, Berget SM: Regulation of alternative splicing by the ATP-dependent DEADbox RNA helicase p72. Mol Cell Biol 2002, 22(16):5698-5707.

10. Li J, Hawkins IC, Harvey CD, Jennings JL, Link AJ, Patton JG: Regulation of alternative splicing by SRrp86 and its interacting proteins. Mol Cell Biol 2003, 23(2I):7437-7447.

11. Rossow KL, Janknecht R: Synergism between p68 RNA helicase and the transcriptional coactivators CBP and $\mathrm{p} 300$. Oncogene 2003, 22(I): $151-156$.

12. Warner DR, Bhattacherjee V, Yin X, Singh S, Mukhopadhyay P, Pisano MM, Greene RM: Functional interaction between Smad, CREB binding protein, and p68 RNA helicase. Biochem Biophys Res Commun 2004, 324(I):70-76.

13. Nakajima T, Uchida C, Anderson SF, Lee CG, Hurwitz J, Parvin JD, Montminy M: RNA helicase A mediates association of CBP with RNA polymerase II. Cell 1997, 90(6): I I07-IIII2.

14. Causevic M, Hislop RG, Kernohan NM, Carey FA, Kay RA, Steele RJ, Fuller-Pace FV: Overexpression and poly-ubiquitylation of the DEAD-box RNA helicase $\mathbf{6 8}$ in colorectal tumours. Oncogene 200I, 20(53):7734-7743.

15. Jacobs AM, Nicol SM, Hislop RG, Jaffray EG, Hay RT, Fuller-Pace FV: SUMO modification of the DEAD box protein p68 modulates its transcriptional activity and promotes its interaction with HDACI. Oncogene 2007, 26(40):5866-5876.

16. Gregory RI, Yan KP, Amuthan G, Chendrimada T, Doratotaj B, Cooch N, Shiekhattar R: The Microprocessor complex mediates the genesis of microRNAs. Nature 2004, 432(70I4):235-240.

17. Merz C, Urlaub H, Will CL, Luhrmann R: Protein composition of human $m R N P s$ spliced in vitro and differential requirements for mRNP protein recruitment. Rna 2007, I3(I): I | 6-I 28.

18. Alpatov R, Munguba GC, Caton P, Joo JH, Shi Y, Shi Y, Hunt ME, Sugrue SP: Nuclear speckle-associated protein Pnn/DRS binds to the transcriptional corepressor CtBP and relieves CtBPmediated repression of the E-cadherin gene. Mol Cell Biol 2004, 24(23): 10223-10235.

19. Moumen A, Masterson P, O'Connor MJ, Jackson SP: hnRNP K: an HDM2 target and transcriptional coactivator of p53 in response to DNA damage. Cell 2005, I23(6): 1065-1078.

20. Kino Y, Mori D, Oma Y, Takeshita Y, Sasagawa N, Ishiura S: Muscleblind protein, MBNLI/EXP, binds specifically to CHHG repeats. Hum Mol Genet 2004, I3(5):495-507.

21. Emili A, Shales M, McCracken S, Xie W, Tucker PW, Kobayashi R, Blencowe BJ, Ingles CJ: Splicing and transcription-associated proteins PSF and $\mathrm{p} 54 \mathrm{nrb} / \mathrm{nonO}$ bind to the RNA polymerase II CTD. Rna 2002, 8(9): | | 02-I| II.

22. Zhang C, Dowd DR, Staal A, Gu C, Lian JB, van Wijnen AJ, Stein GS, MacDonald PN: Nuclear coactivator-62 kDa/Ski-interacting protein is a nuclear matrix-associated coactivator that may couple vitamin D receptor-mediated transcription and RNA splicing. I Biol Chem 2003, 278(37):35325-35336.

23. Liang S, Lutz CS: p54nrb is a component of the snRNP-free UIA (SF-A) complex that promotes pre-mRNA cleavage during polyadenylation. Rna 2006, I 2(I): III-I2I.

24. Pubmed [http://www.ncbi.nlm.nih.gov/sites/entrez?db=pubmed]

25. Fatigo [http://fatigo.bioinfo.cipf.es/]

26. Genecards [http://www.genecards.org]

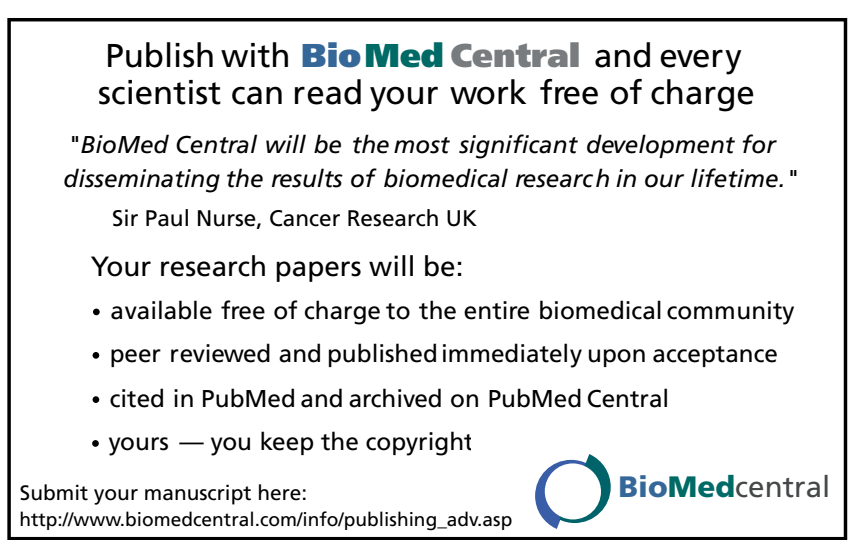

